\title{
Erratum to: Severe Drug-Induced Skin and Liver Injury from Rivaroxaban
}

\author{
Patrick Barrett $\cdot$ Raj Vuppalanchi • \\ Howard Masuoka $\cdot$ Naga Chalasani
}

Published online: 13 February 2015

(c) Springer Science+Business Media New York 2015

\section{Erratum to: Dig Dis Sci \\ DOI 10.1007/s10620-014-3504-9}

The original version of this article unfortunately contained a mistake. The presentation of the figure legend was incorrect.

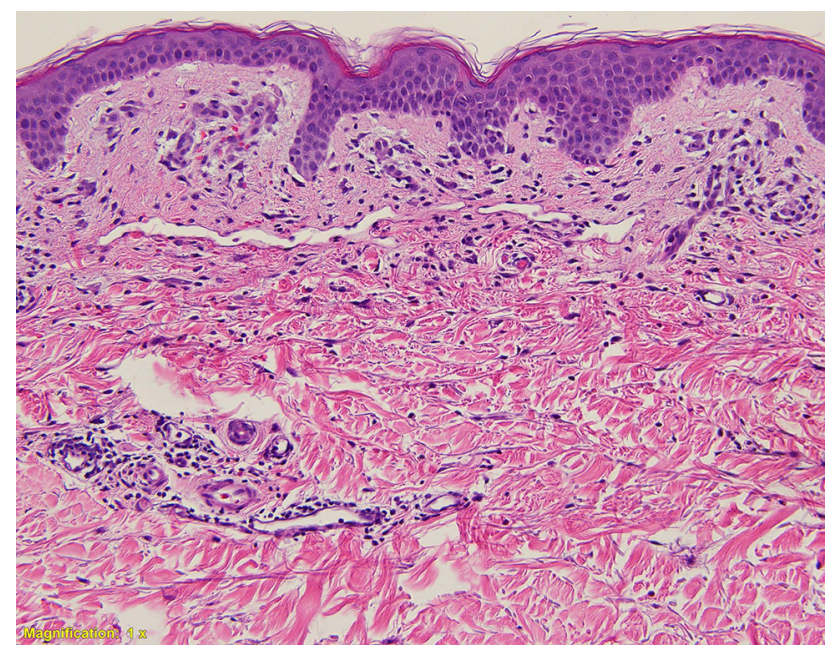

Fig. 3 Skin biopsy taken from the right axilla demonstrates a superficial dermal perivascular infiltrate composed of lymphocytes and eosinophils with neutrophil vascular margination (hematoxylin and eosin at $\times 1$ magnification)
The legends to Figs. 3 and 4 were interchanged. The correct versions are given below.

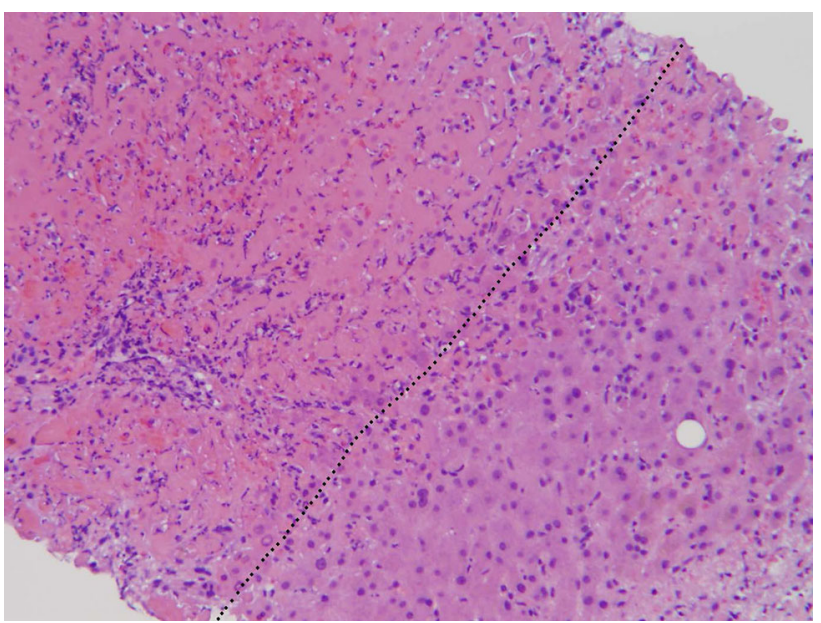

Fig. 4 Liver biopsy showing confluent area of necrosis (left of dotted line) containing a neutrophilic inflammatory infiltrate (hematoxylin and eosin at $\times 10$ magnification)
The online version of the original article can be found under doi:10.1007/s10620-014-3504-9.

P. Barrett · R. Vuppalanchi $(\bowtie) \cdot$ H. Masuoka $\cdot$ N. Chalasani Division of Gastroenterology and Hepatology, Department of Medicine, Indiana University School of Medicine, 702 Rotary Building, Suite 225, Indianapolis, IN 46202, USA

e-mail: rvuppala@iupui.edu 